\title{
Multiple solutions for $p$-Laplacian systems with critical homogeneous nonlinearity
}

\author{
Dengfeng Lü
}

\section{Correspondence:}

dengfeng1214@163.com

School of Mathematics and

Statistics, Hubei Engineering

University, Hubei 432000, P. R.

China

\section{Abstract}

In this article, we deal with existence and multiplicity of solutions to the $p$-Laplacian system of the type

$$
\begin{cases}-\Delta_{p} u=\frac{1}{p^{*}} \frac{\partial F(x, u, v)}{\partial u}+\lambda|u|^{q-2} u, & x \in \Omega, \\ -\Delta_{p} v=\frac{1}{p^{*}} \frac{\partial F(x, u, v)}{\partial v}+\delta|v|^{q-2} v, & x \in \Omega, \\ u=v=0, & x \in \partial \Omega,\end{cases}
$$

where $\Omega \subset \mathbb{R}^{N}$ is a bounded domain with smooth boundary $\partial \Omega, \Delta_{p} u=\operatorname{div}\left(|\nabla u|^{p-2} \nabla u\right)$ is the $p$-Laplacian operator, $N \geq p^{2}, 2 \leq p \leq q<p^{*}, p^{*}=\frac{N p}{N-p}$ denotes the Sobolev critical exponent, $F \in C^{1}\left(\bar{\Omega} \times \mathbb{R}^{+} \times \mathbb{R}^{+}, \mathbb{R}^{+}\right)$is a homogeneous function of degree $p^{*}$. By using the variational method and Ljusternik-Schnirelmann theory, we prove that the system has at least $\operatorname{cat}_{\Omega}(\Omega)$ distinct nonnegative solutions.

AMS 2010 Mathematics Subject Classifications: 35J50; 35B33.

Keywords: $p$-Laplacian system, Ljusternik-Schnirelmann theory, critical exponent, multiple solutions

\section{Introduction and main results}

In this article, we consider the existence and multiplicity of solutions for the following critical $p$-Laplacian system:

$$
\begin{cases}-\Delta_{p} u=\frac{1}{p^{*}} \frac{\partial F(x, u, v)}{\partial u}+\lambda|u|^{q-2} u, & x \in \Omega \\ -\Delta_{p} v=\frac{1}{p^{*}} \frac{\partial F(x, u, v)}{\partial v}+\delta|v|^{q-2} v, & x \in \Omega \\ u=v=0, & x \in \partial \Omega\end{cases}
$$

where $\Omega \subset \mathbb{R}^{N}$ is a bounded domain with smooth boundary $\partial \Omega, \Delta_{p} u=\operatorname{div}\left(|\nabla u|^{p-2} \nabla u\right)$ is the $p$-Laplacian operator, $N \geq p^{2}, 2 \leq p \leq q<p^{*}, p^{*}=\frac{N p}{N-p}$ denotes the Sobolev critical exponent, $F \in C^{1}\left(\bar{\Omega} \times \mathbb{R}^{+} \times \mathbb{R}^{+}, \mathbb{R}^{+}\right)$is a homogeneous function of degree $p^{*},\left(\frac{\partial F(x, u, v)}{\partial u}, \frac{\partial F(x, u, v)}{\partial v}\right)=\nabla F$ and $\lambda, \delta$ are positive parameters. 
The starting point on the study of the system (1.1) is its scalar version:

$$
\left\{\begin{array}{lr}
-\Delta_{p} u=|u|^{p^{*}-2} u+\lambda|u|^{q-2} u, & x \in \Omega \\
u=0, & x \in \partial \Omega,
\end{array}\right.
$$

with $2 \leq p \leq q<p^{*}$. In a pioneer work Brezis and Nirenberg [1] showed that, if $p=q$ $=2$, the equation (1.2) has at least one positive solution provided $N \geq 4$ and $0<\lambda<\lambda_{1}$, where $\lambda_{1}$ is the first eigenvalue of the operator $\left(-\Delta, H_{0}^{1}(\Omega)\right)$. In particular, the first multiplicity result for (1.2) has been achieved by Rey [2] in the semilinear case. Precisely Rey proved that if $N \geq 5, p=q=2$, for $\lambda$ small enough equation (1.2) has at least $\operatorname{cat}_{\Omega}(\Omega)$ solutions, where $\operatorname{cat}_{\Omega}(\Omega)$ denotes the Ljusternik-Schnirelmann category of $\Omega$ in itself. Furthermore, Alves and Ding [3] obtained the existence of cat ${ }_{\Omega}(\Omega)$ positive solutions to equation (1.2) with $p \geq 2, p \leq q<p^{*}$.

In recent years, more and more attention have been paid to the elliptic systems. In particular, Ding and Xiao [4] concerned the case $F(x, u, v)=2|u|^{\alpha}|v|^{\beta}, \alpha>1, \beta>1$ satisfying $\alpha+\beta=p^{*}$, i.e., the following elliptic system

$$
\begin{cases}-\Delta_{p} u=\frac{2 \alpha}{\alpha+\beta}|u|^{\alpha-2} u|v|^{\beta}+\lambda|u|^{q-2} u, & x \in \Omega, \\ -\Delta_{p} v=\frac{2 \beta}{\alpha+\beta}|u|^{\alpha}|v|^{\beta-2} v+\delta|v|^{q-2} v, & x \in \Omega, \\ u=v=0, & x \in \partial \Omega .\end{cases}
$$

Using standard tools of the variational theory and the Ljusternik-Schnirelmann category theory, Ding and Xiao [4] have proved that system (1.3) has at least cat ${ }_{\Omega}(\Omega)$ positive solutions if $\lambda, \delta$ satisfied a certain condition. Hsu [5] obtained the existence of two positive solutions of system (1.3) with the sublinear perturbation of $1<q<p<N$. Recently, Shen and Zhang [6] extended the results in [5] to the case (1.1) with $1<q<p$ $<N$ and obtained similar results. In this article, we study (1.1) and complement the results of $[5,6]$ to the case $2 \leq p \leq q<p^{*}$, also extend the results of $[4,7]$. To the best of our knowledge, problem (1.1) has not been considered before. Thus it is necessary for us to investigate the critical $p$-Laplacian systems (1.1) deeply. For more similar problems, we refer to [8-17], and references therein.

Before stating our results, we need the following assumptions:

$\left(F_{0}\right) \quad F \in C^{1}\left(\bar{\Omega} \times \mathbb{R}^{+} \times \mathbb{R}^{+}, \mathbb{R}^{+}\right)$and $F(x, t u, t v)=t^{p^{*}} F(x, u, v)(t>0)$ holds for all $(x, u, v) \in \bar{\Omega} \times \mathbb{R}^{+} \times \mathbb{R}^{+}$;

$\left(F_{1}\right) F(x, u, 0)=F(x, 0, v)=\frac{\partial F(x, u, 0)}{\partial u}=\frac{\partial F(x, 0, v)}{\partial v}=0$, where $u, v \in \mathbb{R}^{+}$;

$\left(F_{2}\right) \frac{\partial F(x, u, v)}{\partial u}, \frac{\partial F(x, u, v)}{\partial v}$ are strictly increasing functions about $u$ and $v$ for all $u, v>0$.

The main results we get are the following:

Theorem 1.1. Suppose $N \geq p^{2}$ and $F$ satisfies $\left(F_{0}\right)-\left(F_{2}\right)$, then the problem (1.1) has at least one nonnegative solution for $2 \leq p<q<p^{*}$ and $\lambda, \delta>0$, or $q=p$ and $\lambda, \delta \in(0$, $\left.\Lambda_{1}\right)$, where $\Lambda_{1}$ is the first eigenvalue of $\left(-\Delta_{p}, W_{0}^{1, p}(\Omega)\right)$.

Theorem 1.2. Suppose $N \geq p^{2}, 2 \leq p \leq q<p *$ and F satisfies $\left(F_{0}\right)-\left(F_{2}\right)$, then there exists $\Lambda>0$ such that the problem (1.1) has at least cat $_{\Omega}(\Omega)$ distinct nonnegative solutions for $\lambda, \delta \in(0, \Lambda)$. 
Remark 1.1. Theorem 1 in [4] is the special case of our Theorem 1.2 corresponding to $F(x, u, v)=2|u|^{\alpha}|v|^{\beta}, \alpha>1, \beta>1, \alpha+\beta=p^{*}$. There are functions $F(x, u, v)$ satisfying the conditions of our Theorems 1.1 and 1.2. Some typical examples are:

(i) $F(x, u, v)=\sum_{i=1}^{k} f_{i}(x)|u|^{\alpha_{i}}|v|^{\beta_{i}}$;

(ii) $F(x, u, v)=\left\{\begin{array}{l}f_{1}(x)|u|^{\frac{3}{2}}|v|^{\frac{5}{2}}+f_{2}(x) \frac{u^{3} v^{3}}{u^{2}+v^{2}}, \quad(u, v) \neq(0,0), \\ 0, \quad(u, v)=(0,0)\end{array}\right.$

where $f_{i}(x) \geq 0, f_{i}(x) \not \equiv 0, f_{i}(x) \in C(\bar{\Omega}) \cap L^{\infty}(\Omega), \alpha_{i}, \beta_{i}>1, \alpha_{i}+\beta_{i}=p^{*}$. Obviously, $F(x$, $u, v)$ satisfies $\left(F_{0}\right)-\left(F_{2}\right)$.

This article is organized as follows. In Section 2, some notations and the MountainPass levels are established and the Theorem 1.1 is proved. We present some technical lemmas which are crucial in the proof of the Theorem 1.2 in Section 3. Theorem 1.2 is proved in Section 4.

\section{Notations and proof of Theorem 1.1}

Throughout this article, $C, C_{i}$ will denote various positive constants whose exact values are not important, $\rightarrow$ (respectively $\rightarrow$ ) denotes strong (respectively weak) convergence. $O\left(\varepsilon^{t}\right)$ denotes $\left|O\left(\varepsilon^{t}\right)\right| / \varepsilon^{t} \leq C$, $o_{m}(1)$ denotes $o_{m}(1) \rightarrow 0$ as $m \rightarrow \infty$. $L^{s}(\Omega)(1 \leq s<+\infty)$ denotes Lebesgue spaces, the norm $L^{s}$ is denoted by $|\cdot|_{s}$ for $1 \leq s<+\infty$. Let $B_{r}(x)$ denotes a ball centered at $x$ with radius $r$, the dual space of a Banach space $E$ will be denoted by $E^{-1}$. We define the product space $E:=W_{0}^{1, p}(\Omega) \times W_{0}^{1, p}(\Omega)$ endowed with the norm $\|(u, v)\|_{E}=\left(\|u\|_{W_{0}^{1, p}(\Omega)}^{p}+\|v\|_{W_{0}^{1, p}(\Omega)}^{p}\right)^{\frac{1}{p}}, \quad$ and $\quad$ the norm $\|u\|_{W_{0}^{1, p}(\Omega)}=\left(\int_{\Omega}|\nabla u|^{p} d x\right)^{\frac{1}{p}}$

Using assumption of $\left(F_{1}\right)$, we have the so-called Euler identity

$$
\frac{\partial F(x, u, v)}{\partial u} u+\frac{\partial F(x, u, v)}{\partial v} v=p^{*} F(x, u, v) .
$$

In addition, we can extend the function $F(x, u, v)$ to the whole $\bar{\Omega} \times \mathbb{R}^{2}$ by considering $\tilde{F}(x, u, v)=F\left(x, u^{+}, v^{+}\right)$, where $u^{+}=\max \{u, 0\}$. It is easy to check that $\tilde{F}(x, u, v)$ is of class $C^{1}$ and its restriction to $\bar{\Omega} \times \mathbb{R}^{+} \times \mathbb{R}^{+}$coincides with $F(x, u, v)$. In order to simplify the notation we shall write, from now on, only $F(x, u, v)$ to denote the above extension.

A pair of functions $(u, v) \in E$ is said to be a weak solution of problem (1.1) if

$$
\begin{gathered}
\int_{\Omega}\left(|\nabla u|^{p-2} \nabla u \nabla \varphi_{1}+|\nabla v|^{p-2} \nabla v \nabla \varphi_{2}\right) d x-\frac{1}{p^{*}} \int_{\Omega}\left(\frac{\partial F(x, u, v)}{\partial u} \varphi_{1}+\frac{\partial F(x, u, v)}{\partial v} \varphi_{2}\right) d x \\
-\int_{\Omega}\left(\lambda|u|^{q-2} u \varphi_{1}+\delta|v|^{q-2} v \varphi_{2}\right) d x=0, \quad \forall\left(\varphi_{1}, \varphi_{2}\right) \in E .
\end{gathered}
$$

Thus, by (2.1) the corresponding energy functional of problem (1.1) is defined on $E$ by

$$
I_{\lambda, \delta}(u, v)=\frac{1}{p} \int_{\Omega}\left(|\nabla u|^{p}+|\nabla v|^{p}\right) d x-\frac{1}{p^{*}} \int_{\Omega} F(x, u, v) d x-\frac{1}{q} \int_{\Omega}\left(\lambda|u|^{q}+\delta|v|^{q}\right) d x .
$$


Using $\left(F_{0}\right)-\left(F_{2}\right)$, we can verify $I_{\lambda, \delta}(u, v) \in C^{1}(E, \mathbb{R})$ (see [6]). It is well known that the weak solutions of problem (1.1) are the critical points of the energy functional $I_{\lambda, \delta}(u$, v).

The functional $I \in C^{1}(E, \mathbb{R})$ is said to satisfy the $(P S)_{c}$ condition if any sequence $\left\{u_{m}\right\}$ $\subset E$ such that as $m \rightarrow \infty, I\left(u_{m}\right) \rightarrow c, I\left(u_{m}\right) \rightarrow 0$ strongly in $E^{-1}$ contains a subsequence converging in $E$ to a critical point of $I$. In this article, we will take $I=I_{\lambda}, \delta(u, v)$ and $E:=W_{0}^{1, p}(\Omega) \times W_{0}^{1, p}(\Omega)$.

As the energy functional $I_{\lambda, \delta}$ is not bounded below on $E$, we need to study $I_{\lambda, \delta}$ on the Nehari manifold

$$
\mathcal{N}_{\lambda, \delta}=\left\{(u, v) \in E \backslash\{(0,0)\}:\left\langle I_{\lambda, \delta}^{\prime}(u, v),(u, v)\right\rangle=0\right\} .
$$

Note that $\mathcal{N}_{\lambda, \delta}$ contains every nonzero solution of problem (1.1), and define the $\operatorname{minimax} c_{\lambda, \delta}$ as

$$
c_{\lambda, \delta}=\inf _{(u, v) \in \mathcal{N}_{\lambda, \delta}} I_{\lambda, \delta}(u, v)
$$

Next, we present some properties of $c_{\lambda, \delta}$ and $\mathcal{N}_{\lambda, \delta}$. Its proofs can be done as [18, Theorem 4.2]. First of all, we note that there exists $\rho>0$, such that

$$
\|(u, v)\|_{E} \geq \rho>0, \quad \forall(u, v) \in \mathcal{N}_{\lambda, \delta} .
$$

It is standard to check that $I_{\lambda, \delta}$ satisfies Mountain-Pass geometry, so we can use the homogeneity of $F$ to prove that $c_{\lambda, \delta}$ can be alternatively characterized by

$$
c_{\lambda, \delta}=\inf _{\gamma \in \Gamma} \max _{t \in[0,1]} I_{\lambda, \delta}(\gamma(t))=\inf _{(u, v) \in E \backslash\{(0,0)\}} \max _{t \geq 0} I_{\lambda, \delta}(t(u, v))>0,
$$

where $\Gamma=\left\{\gamma \in C([0,1], E): \gamma(0)=0, I_{\lambda, \delta}(\gamma(1))<0\right\}$. Moreover, for each $(u, v) \in E$ $\backslash\{(0,0)\}$, there exists a unique $t^{*}>0$ such that $t^{*}(u, v) \in \mathcal{N}_{\lambda, \delta}$. The maximum of the function $t \mapsto I_{\lambda, \delta}(t(u, v))$, for $t \geq 0$, is achieved at $t=t^{*}$.

In this section, we will find the range of $c$ where the $(P S)_{c}$ condition holds for the functional $I_{\lambda, \delta}$. First let us define

$$
S_{F}=\inf _{(u, v) \in E \backslash\{(0,0)\}}\left\{\frac{\int_{\Omega}|\nabla u|^{p}+|\nabla v|^{p} d x}{\left(\int_{\Omega} F(x, u, v) d x\right)^{\frac{p}{p^{*}}}}: \int_{\Omega} F(x, u, v) d x>0\right\} .
$$

Lemma 2.1. If $N \geq p^{2}$ and $F$ satisfies $\left(F_{0}\right)-\left(F_{2}\right)$, then the functional $I_{\lambda, \delta}$ satisfies the $(P S)_{c}$ condition for all $c<\frac{1}{N} S_{F}^{\frac{N}{p}}$, provide one of the following conditions holds

(i) $2 \leq p<q<p^{*}$ and $\lambda, \delta>0$;

(ii) $q=p$, and $\lambda, \delta \in\left(0, \Lambda_{1}\right)$, where $\Lambda_{1}>0$ denotes the first eigenvalue of $\left(-\Delta_{p}, W_{0}^{1, p}(\Omega)\right)$.

Proof. Let $\left\{\left(u_{m}, v_{m}\right)\right\} \subset E$ such that $I_{\lambda, \delta}^{\prime}\left(u_{m}, v_{m}\right) \rightarrow 0$ and $I_{\lambda, \delta}\left(u_{m}, v_{m}\right) \rightarrow c<\frac{1}{N} S_{F}^{\frac{N}{p}}$. Now, we first prove that $\left\{\left(u_{m}, v_{m}\right)\right\}$ is bounded in $E$. If the above item (i) is true it suffices to use the definition of $I_{\lambda, \delta}$ to obtain $C_{1}>0$ such that 


$$
\begin{aligned}
c+C_{1}\left\|\left(u_{m}, v_{m}\right)\right\|_{E}+o_{m}(1) & \geq I_{\lambda, \delta}\left(u_{m}, v_{m}\right)-\frac{1}{q}\left\langle I_{\lambda, \delta}^{\prime}\left(u_{m}, v_{m}\right),\left(u_{m}, v_{m}\right)\right\rangle \\
& =\left(\frac{1}{p}-\frac{1}{q}\right)\left\|\left(u_{m}, v_{m}\right)\right\|_{E}^{p}+\left(\frac{1}{q}-\frac{1}{p^{*}}\right) \int_{\Omega} F\left(x, u_{m}, v_{m}\right) d x \\
& \geq \frac{q-p}{p q}\left\|\left(u_{m}, v_{m}\right)\right\|_{E}^{p} .
\end{aligned}
$$

The above expression implies that $\left\{\left(u_{m}, v_{m}\right)\right\} \subset E$ is bounded. When (ii) occurs, in this case, it follows that

$$
\int_{\Omega}\left(\lambda\left|u_{m}\right|^{p}+\delta\left|v_{m}\right|^{p}\right) d x \leq \max \{\lambda, \delta\} \int_{\Omega}\left(\left|u_{m}\right|^{p}+\left|v_{m}\right|^{p}\right) d x \leq \frac{\max \{\lambda, \delta\}}{\Lambda_{1}}\left\|\left(u_{m}, v_{m}\right)\right\|_{E^{\prime}}^{p}
$$

and therefore we get

$$
\begin{aligned}
c+C_{1}\left\|\left(u_{m}, v_{m}\right)\right\|_{E}+o_{m}(1) & \geq I_{\lambda, \delta}\left(u_{m}, v_{m}\right)-\frac{1}{p^{*}}\left\langle I_{\lambda, \delta}^{\prime}\left(u_{m}, v_{m}\right),\left(u_{m}, v_{m}\right)\right\rangle \\
& =\left(\frac{1}{p}-\frac{1}{p^{*}}\right)\left\|\left(u_{m}, v_{m}\right)\right\|_{E}^{p}+\left(\frac{1}{p^{*}}-\frac{1}{p}\right) \int_{\Omega}\left(\lambda\left|u_{m}\right|^{p}+\delta\left|v_{m}\right|^{p}\right) d x \\
& \geq \frac{1}{N}\left(1-\frac{\max \{\lambda, \delta\}}{\Lambda_{1}}\right)\left\|\left(u_{m}, v_{m}\right)\right\|_{E}^{p} .
\end{aligned}
$$

Since $\lambda, \delta \in\left(0, \Lambda_{1}\right)$ the boundedness of $\left\{\left(u_{m}, v_{m}\right)\right\}$ follows as the first case.

So, $\left\{\left(u_{m}, v_{m}\right)\right\}$ is bounded in $E$. Going if necessary to a subsequence, we can assume that

$$
\left\{\begin{array}{l}
\left(u_{m}, v_{m}\right) \rightarrow(u, v), \text { in } E \\
\left(u_{m}, v_{m}\right) \rightarrow(u, v), \text { a.e. in } \Omega \\
\left(u_{m}, v_{m}\right) \rightarrow(u, v), \text { in } L^{s}(\Omega) \times L^{s}(\Omega), 1 \leq s<p^{*}
\end{array}\right.
$$

as $m \rightarrow \infty$. Clearly, we have

$$
\int_{\Omega}\left(\lambda\left|u_{m}\right|^{q}+\delta\left|v_{m}\right|^{q}\right) d x=\int_{\Omega}\left(\lambda|u|^{q}+\delta|v|^{q}\right) d x+o_{m}(1) .
$$

Moreover, a standard argument shows that $I_{\lambda, \delta}^{\prime}(u, v)=0$. Thus we get

$$
\begin{aligned}
I_{\lambda, \delta}(u, v) & =\frac{1}{p}\|(u, v)\|_{E}^{p}-\frac{1}{p^{*}} \int_{\Omega} F(x, u, v) d x-\frac{1}{q} \int_{\Omega}\left(\lambda|u|^{q}+\delta|v|^{q}\right) d x \\
& =\left(\frac{1}{p}-\frac{1}{q}\right)\|(u, v)\|_{E}^{p}+\left(\frac{1}{q}-\frac{1}{p^{*}}\right) \int_{\Omega} F(x, u, v) d x \\
& \geq 0
\end{aligned}
$$

Let $\left(\tilde{u}_{m}, \tilde{v}_{m}\right)=\left(u_{m}-u, v_{m}-v\right)$, then by Brezis-Lieb Lemma in [19] implies

$$
\left\|\left(u_{m}, v_{m}\right)\right\|_{E}^{p}=\|(u, v)\|_{E}^{p}+\left\|\left(\tilde{u}_{m}, \tilde{v}_{m}\right)\right\|_{E}^{p}+o_{m}(1)
$$

By the same method of [8, Lemma 5] (or [6, Lemma 3.4]), we obtain

$$
\int_{\Omega} F\left(x, u_{m}, v_{m}\right) d x=\int_{\Omega} F(x, u, v) d x+\int_{\Omega} F\left(x, \tilde{u}_{m}, \tilde{v}_{m}\right) d x+o_{m}(1) .
$$


By (2.4)-(2.7) and the weak convergence of $\left(u_{m}, v_{m}\right)$, we have

$$
\begin{aligned}
c+o_{m}(1) & =I_{\lambda, \delta}(u, v)+\frac{1}{p}\left\|\left(\tilde{u}_{m}, \tilde{v}_{m}\right)\right\|_{E}^{p}-\frac{1}{p^{*}} \int_{\Omega} F\left(x, \tilde{u}_{m}, \tilde{v}_{m}\right) d x \\
& \geq \frac{1}{p}\left\|\left(\tilde{u}_{m}, \tilde{v}_{m}\right)\right\|_{E}^{p}-\frac{1}{p^{*}} \int_{\Omega} F\left(x, \tilde{u}_{m}, \tilde{v}_{m}\right) d x .
\end{aligned}
$$

By using $I_{\lambda, \delta}^{\prime}\left(u_{m}, v_{m}\right) \rightarrow 0$ and (2.4), (2.6), and (2.7), we get

$$
\begin{aligned}
o_{m}(1) & =\left\langle I_{\lambda, \delta}^{\prime}\left(u_{m}, v_{m}\right),\left(u_{m}, v_{m}\right)\right\rangle \\
& =\left\|\left(u_{m}, v_{m}\right)\right\|_{E}^{p}-\int_{\Omega} F\left(x, u_{m}, v_{m}\right) d x-\int_{\Omega}\left(\lambda\left|u_{m}\right|^{q}+\delta\left|v_{m}\right|^{q}\right) d x \\
& =\left\langle I_{\lambda, \delta}^{\prime}(u, v),(u, v)\right\rangle+\left\|\left(\tilde{u}_{m}, \tilde{v}_{m}\right)\right\|_{E}^{p}-\int_{\Omega} F\left(x, \tilde{u}_{m}, \tilde{v}_{m}\right) d x .
\end{aligned}
$$

Recalling that $I_{\lambda, \delta}^{\prime}(u, v)=0$, we can use the above equality and (2.8) to obtain

$$
\lim _{m \rightarrow \infty}\left\|\left(\tilde{u}_{m}, \tilde{v}_{m}\right)\right\|_{E}^{p}=k=\lim _{m \rightarrow \infty} \int_{\Omega} F\left(x, \tilde{u}_{m}, \tilde{v}_{m}\right) d x, \quad c \geq\left(\frac{1}{p}-\frac{1}{p^{*}}\right) k=\frac{1}{N} k,
$$

where $k$ is a nonnegative number.

In view of the definition of $S_{F}$, we have that

$$
\left\|\left(\tilde{u}_{m}, \tilde{v}_{m}\right)\right\|_{E}^{p} \geq S_{F}\left(\int_{\Omega} F\left(x, \tilde{u}_{m}, \tilde{v}_{m}\right) d x\right)^{\frac{p}{p^{*}}} .
$$

Taking the limit we get $k \geq S_{F} k^{\frac{p}{p *}}$. So, if $k>0$, we conclude that $k \geq S_{F}^{\frac{N}{p}}$ and therefore

$$
\frac{1}{N} S_{F}^{\frac{N}{p}} \leq \frac{1}{N} k \leq c<\frac{1}{N} S_{F}^{\frac{N}{p}}
$$

which is a contradiction. Hence $k=0$ and therefore $\left(u_{m}, v_{m}\right) \rightarrow(u, v)$ strongly in $E$. Before presenting our next result we recall that, for each $\varepsilon>0$, the function

$$
U_{\varepsilon}(x)=\frac{C_{N} \cdot \varepsilon^{\frac{N-p}{p^{2}}}}{\left(\varepsilon+|x|^{\frac{p}{p-1}}\right)^{\frac{N-p}{p}}}, \quad C_{N}=\left(N\left(\frac{N-p}{p-1}\right)^{p-1}\right)^{\frac{N-p}{p^{2}}}, \quad x \in \mathbb{R}^{N}
$$

satisfies

$$
\left|\nabla U_{\varepsilon}(x)\right|_{p}^{p}=\left|U_{\varepsilon}(x)\right|_{p^{*}}^{p^{*}}=S^{\frac{N}{p}}
$$

where $S$ is the best constant of the Sobolev embedding $D^{1, p}\left(\mathbb{R}^{N}\right) \hookrightarrow L^{p^{*}}\left(\mathbb{R}^{N}\right)$. Thus, using [8, Lemma 3] and the homogeneity of $F$, we obtain $A, B>0$ such that 


$$
S_{F}=\frac{\left\|\left(A U_{\varepsilon}, B U_{\varepsilon}\right)\right\|_{E}^{p}}{\left(\int_{\mathbb{R}^{N}} F\left(x, A U_{\varepsilon} B U_{e}\right) d x\right)^{\frac{p}{p^{*}}}}=\frac{A^{p}+B^{p}}{(F(x, A, B))^{\frac{p}{p^{*}}}} \cdot \frac{S^{\frac{N}{p}}}{\left|U_{\varepsilon}\right|_{p^{*}}^{p^{*}}},
$$

from which and (2.10) it follows that

$$
S_{F}=\frac{A^{p}+B^{p}}{(F(x, A, B))^{\frac{p}{p^{*}}}} S .
$$

We define a cut-off function $\phi(x) \in C_{0}^{\infty}\left(\mathbb{R}^{N}\right)$ such that $\varphi(x)=1$ if $|x| \leq R ; \varphi(x)=0$ if $|x| \geq 2 R$ and $0 \leq \varphi(x) \leq 1$, where $B_{2 R}(0) \subset \Omega$, set $u_{\varepsilon}=\frac{\phi(x) U_{\varepsilon}}{\left|\phi U_{\varepsilon}\right|_{p^{*}}}$, where $U_{\varepsilon}$ was defined in (2.9). So that $\left|u_{\varepsilon}\right|_{p^{*}}=1$. Then, we can get the following results from [[20], Lemma 11.1]:

$$
\begin{aligned}
& \left\|u_{\varepsilon}\right\|_{W_{0}^{1, p}(\Omega)}^{p}=S+O\left(\varepsilon^{\frac{N-p}{p}}\right), \\
& \int_{\Omega}\left|u_{\varepsilon}\right|^{\xi} d x \approx \begin{cases}\varepsilon^{\frac{N-p}{p^{2} \xi},} & \text { if } 1<\xi<p^{*}\left(1-\frac{1}{p}\right), \\
\varepsilon^{\frac{N-p}{p^{2}}}|\ln \varepsilon|, & \text { if } \xi=p^{*}\left(1-\frac{1}{p}\right), \\
\varepsilon \frac{(p-1)(N p-\xi(N-p))}{p^{2}}, & \text { if } p^{*}\left(1-\frac{1}{p}\right)<\xi<p^{*},\end{cases}
\end{aligned}
$$

where $A \approx B$ means $C_{1} B \leq A \leq C_{2} B$.

Lemma 2.2. Suppose that $F$ satisfies $\left(F_{0}\right)-\left(F_{2}\right), 2 \leq p<q<p^{*}$ and $\lambda>0, \delta>0$, then $c_{\lambda, \delta}<\frac{1}{N} S_{F}^{\frac{N}{p}}$. The same result holds if $q=p$ and $\lambda, \delta \in\left(0, \Lambda_{1}\right)$, where $\Lambda_{1}>0$ denotes the first eigenvalue of $\left(-\Delta_{p}, W_{0}^{1, p}(\Omega)\right)$.

Proof. We can use the homogeneity of $F$ to get, for any $t \geq 0$,

$$
I_{\lambda, \delta}\left(t A u_{\varepsilon}, t B u_{\varepsilon}\right)=\frac{t^{p}}{p}\left(A^{p}+B^{p}\right)\left\|u_{\varepsilon}\right\|_{W_{0}^{1, p}(\Omega)}^{p}-\frac{t^{p^{*}}}{p^{*}} F(x, A, B)-\frac{t^{q}}{q}\left(\lambda A^{q}+\delta B^{q}\right)\left|u_{\varepsilon}\right|_{q}^{q} .
$$

We shall denote by $h(t)$ the right-hand side of the above equality and consider two distinct cases.

Case 1. $2 \leq p<q<p^{*}$.

From the fact that $\lim _{t \rightarrow+\infty} h(t)=-\infty$ and $h(t)>0$ when $t$ is close to 0 , there exists $t_{\varepsilon}>$ 0 such that

$$
h\left(t_{\varepsilon}\right)=\max _{t \geq 0} h(t) .
$$

Let

$$
g(t)=\frac{t^{p}}{p}\left(A^{p}+B^{p}\right)\left\|u_{\varepsilon}\right\|_{W_{0}^{1, p}(\Omega)}^{p}-\frac{t^{p^{*}}}{p^{*}} F(x, A, B), \quad t \geq 0,
$$


and notice that the maximum value of $g(t)$ occurs at the point

$$
\tilde{t}_{\varepsilon}=\left(\frac{\left(A^{p}+B^{p}\right)\left\|u_{\varepsilon}\right\|_{W_{0}^{1, p}(\Omega)}^{p}}{F(x, A, B)}\right)^{\frac{1}{p^{*}-p}} .
$$

So, for each $t \geq 0$,

$$
g(t) \leq g\left(\tilde{t}_{\varepsilon}\right)=\frac{1}{N}\left(\frac{\left(A^{p}+B^{p}\right)\left\|u_{\varepsilon}\right\|_{W_{0}^{1, p}(\Omega)}^{p}}{(F(x, A, B))^{\frac{p}{p^{*}}}}\right)^{\frac{N}{p}},
$$

and therefore

$$
h\left(t_{\varepsilon}\right) \leq \frac{1}{N}\left(\frac{\left(A^{p}+B^{p}\right)\left\|u_{\varepsilon}\right\|_{W_{0}^{1, p}(\Omega)}^{p}}{(F(x, A, B))^{\frac{p}{p^{*}}}}\right)^{\frac{N}{p}}-\frac{t_{\varepsilon}^{q}}{q}\left(\lambda A^{q}+\delta B^{q}\right)\left|u_{\varepsilon}\right|_{q}^{q} .
$$

We claim that, for some $C_{2}>0$, there holds

$$
t_{\varepsilon}^{q}\left(\lambda A^{q}+\delta B^{q}\right) \geq C_{2} .
$$

Indeed, if this is not the case, we have that $t_{\varepsilon_{m}} \rightarrow 0$ for some sequence $\varepsilon_{m} \rightarrow 0^{+}$, then,

$$
0<c_{\lambda, \delta} \leq \sup _{t \geq 0} I_{\lambda, \delta}\left(t A u_{\varepsilon_{m}}, t B u_{\varepsilon_{m}}\right)=I_{\lambda, \delta}\left(t_{\varepsilon_{m}} A u_{\varepsilon_{m}}, t_{\varepsilon_{m}} B u_{\varepsilon_{m}}\right) \rightarrow 0,
$$

which is a contradiction. So, the claim holds and we infer from (2.15) and (2.11)(2.13) that

$$
\begin{aligned}
h\left(t_{\varepsilon}\right) & \leq \frac{1}{N}\left(\frac{A^{p}+B^{p}}{(F(x, A, B))^{\frac{p}{p^{*}}}}\left(S+O\left(\varepsilon^{\frac{N-p}{p}}\right)\right)\right)^{\frac{N}{p}}-C_{3}\left|u_{\varepsilon}\right|_{q}^{q} \\
& \leq \frac{1}{N} S_{F}^{\frac{N}{p}}+O\left(\varepsilon^{\frac{N-p}{p}}\right)-C_{3}\left|u_{\varepsilon}\right|_{q}^{q} \\
& \leq \frac{1}{N} S_{F}^{\frac{N}{p}}+O\left(\varepsilon^{\frac{N-p}{p}}\right)-O\left(\varepsilon^{\frac{(p-1)(N p-q(N-p))}{p^{2}}}\right),
\end{aligned}
$$

where $C_{3}=\frac{C_{2}}{q}$. We know $p^{*}\left(1-\frac{1}{p}\right) \leq p<q<p^{*}$ if $N \geq p^{2}$. By $N \geq p^{2}$ and $2 \leq p<q$ $<p^{*}$ we obtain $\frac{N-p}{p}>\frac{(p-1)(N p-q(N-p))}{p^{2}}$. Thus from the above inequality we conclude that, for each $\varepsilon>0$ small, there holds

$$
c_{\lambda, \delta} \leq \sup _{t \geq 0} I_{\lambda, \delta}\left(t A u_{\varepsilon_{m}}, t B u_{\varepsilon_{m}}\right)=h\left(t_{\varepsilon}\right)<\frac{1}{N} S_{F}^{\frac{N}{p}} .
$$

Case 2. $q=p$.

In this case, we have that $h^{\prime}(t)=0$ if and only if,

$$
\left(A^{p}+B^{p}\right)\left\|u_{\varepsilon}\right\|_{W_{0}^{1, p}(\Omega)}^{p}-\left(\lambda A^{p}-\delta B^{p}\right)\left|u_{\varepsilon}\right|_{p}^{p}=t^{p *-p} F(x, A, B) .
$$


Since we suppose $\lambda, \delta \in\left(0, \Lambda_{1}\right)$, we can use Poincaré's inequality to obtain

$$
\begin{aligned}
\left(\lambda A^{p}+\delta B^{p}\right)\left|u_{\varepsilon}\right|_{p}^{p} & \leq \max \{\lambda, \delta\}\left(A^{p}+B^{p}\right)\left|u_{\varepsilon}\right|_{p}^{p} \\
& <\Lambda_{1}\left(A^{p}+B^{p}\right)\left|u_{\varepsilon}\right|_{p}^{p} \\
& \leq\left(A^{p}+B^{p}\right)\left\|u_{\varepsilon}\right\|_{W_{0}^{1, p}(\Omega)}^{p} .
\end{aligned}
$$

Thus, there exists $t_{\varepsilon}>0$ satisfying (2.14).

Arguing as in the first case we conclude that, from (2.16) for $\varepsilon>0$ small, there holds

$$
\begin{aligned}
h\left(t_{\varepsilon}\right) & \leq \frac{1}{N} S_{F}^{\frac{N}{p}}+O\left(\varepsilon^{\frac{N-p}{p}}\right)-C_{3}\left|u_{\varepsilon}\right|_{p}^{p} \\
& =\left\{\begin{array}{c}
\frac{1}{N} S_{F}^{\frac{N}{p}}+O\left(\varepsilon^{p-1}\right)-O\left(\varepsilon^{p-1}|\ln \varepsilon|\right), N=p^{2}, \\
\frac{1}{N} S_{F}^{\frac{N}{p}}+O\left(\varepsilon^{\frac{N-p}{p}}\right)-O\left(\varepsilon^{p-1}\right), N>p^{2} .
\end{array}\right.
\end{aligned}
$$

Because $p^{*}\left(1-\frac{1}{p}\right)<p$ if $N>p^{2}$ and $p^{*}\left(1-\frac{1}{p}\right)=p$ if $N=p^{2}$, then $\varepsilon^{p-1}=o\left(\varepsilon^{p-1} \mid \ln \right.$

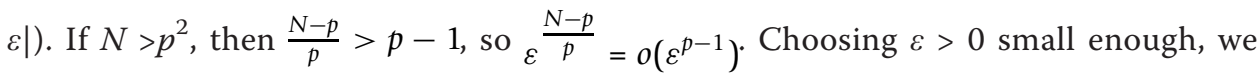
have

$$
c_{\lambda, \delta} \leq \sup _{t \geq 0} I_{\lambda, \delta}\left(t A u_{\varepsilon}, t B u_{\varepsilon}\right)=h\left(t_{\varepsilon}\right)<\frac{1}{N} S_{F}^{\frac{N}{p}} .
$$

This concludes the proof.

By Lemmas 2.1 and 2.2 we can prove our first result.

\section{Proof of Theorem 1.1.}

Since $I_{\lambda, \delta}$ satisfies the geometric conditions of the Mountain-Pass theorem, there exists $\left\{\left(u_{m}, v_{m}\right)\right\} \subset E$ such that $I_{\lambda, \delta}\left(u_{m}, v_{m}\right) \rightarrow c_{\lambda, \delta}, I_{\lambda, \delta}^{\prime}\left(u_{m}, v_{m}\right) \rightarrow 0$. It follows from Lemmas 2.1 and 2.2 that $\left\{\left(u_{m}, v_{m}\right)\right\}$ converges, along a subsequence, to a nonzero critical point $(u, v) \in E$ of $I_{\lambda, \delta}$. Then, if we denote by $u^{-}=\max \{-u, 0\}$ and $v^{-}=\max \{-v, 0\}$ the negative part of $u$ and $v$, respectively, we get

$$
\begin{aligned}
0=\left\langle I_{\lambda, \delta}^{\prime}(u, v),\left(u^{-}, v^{-}\right)\right\rangle= & -\left\|\left(u^{-}, v^{-}\right)\right\|_{E}^{p}-\frac{1}{p^{*}} \int_{\Omega}\left(\frac{\partial F(x, u, v)}{\partial u} u^{-}+\frac{\partial F(x, u, v)}{\partial v} v^{-}\right) d x \\
& -\int_{\Omega}\left(\lambda|u|^{q-2} u u^{-}+\delta|v|^{q-2} v v^{-}\right) d x \\
\leq & -\left\|\left(u^{-}, v^{-}\right)\right\|_{E^{\prime}}^{p}
\end{aligned}
$$

it follows that $\left(u^{-}, v^{-}\right)=(0,0)$. Hence, $u, v \geq 0$ in $\Omega$. The Theorem 1.1 is proved.

We finalize this section with the study of the asymptotic behavior of the minimax level $c_{\lambda, \delta}$ as both the parameters $\lambda, \delta$ approach zero.

Lemma 2.3. $\lim _{\lambda, \delta \rightarrow 0^{+}} c_{\lambda, \delta}=c_{0,0}=\frac{1}{N} S_{F}^{\frac{N}{p}}$.

Proof. We first prove the second equality. It follows from $\lambda=\delta=0$ that $\lambda|u|^{q}+\delta|v|^{q}$ $\equiv 0$. If $A, B, u_{\varepsilon}, g_{\varepsilon}$, and $t_{\varepsilon}$ are the same as those in the proof of Lemma 2.2, we have that $\left(t_{\varepsilon} A u_{\varepsilon}, t_{\varepsilon} B u_{\varepsilon}\right) \in \mathcal{N}_{0,0}$. Thus 


$$
\begin{aligned}
c_{0,0} & \leq I_{0,0}\left(t_{\varepsilon} A u_{\varepsilon}, t_{\varepsilon} B u_{\varepsilon}\right) \\
& =\frac{1}{N}\left(\frac{A^{p}+B^{p}}{(F(x, A, B))^{\frac{p}{p^{*}}}}\left\|u_{\varepsilon}\right\|_{W_{0}^{1, p}(\Omega)}^{p}\right)^{\frac{N}{p}} \\
& =\frac{1}{N}\left(\frac{A^{p}+B^{p}}{(F(x, A, B))^{\frac{p}{p^{*}}}}\left(S+O\left(\varepsilon^{\frac{N-p}{p}}\right)\right)\right)^{\frac{N}{p}} .
\end{aligned}
$$

Taking the limit as $\varepsilon \rightarrow 0^{+}$and using (2.11), we conclude that $c_{0,0} \leq \frac{1}{N} S_{F}^{\frac{N}{p}}$.

In order to obtain the reverse inequality we consider $\left\{\left(u_{m}, v_{m}\right)\right\} \subset E$ such that $I_{0,0}$ $\left(u_{m}, v_{m}\right) \rightarrow c_{0,0}$ and $I_{0,0}^{\prime}\left(u_{m}, v_{m}\right) \rightarrow 0$. It is easy to show that the sequence $\left\{\left(u_{m}, v_{m}\right)\right\}$ is bounded in $E$ and therefore $\left\langle I_{0,0}^{\prime}\left(u_{m}, v_{m}\right),\left(u_{m}, v_{m}\right)\right\rangle=\left\|\left(u_{m}, v_{m}\right)\right\|_{E}^{p}-\int_{\Omega} F\left(x, u_{m}, v_{m}\right) d x=o_{m}(1)$. It follows that

$$
\lim _{m \rightarrow \infty}\left\|\left(u_{m}, v_{m}\right)\right\|_{E}^{p}=l=\lim _{m \rightarrow \infty} \int_{\Omega} F\left(x, u_{m}, v_{m}\right) d x .
$$

Taking the limit in the inequality $S_{F}\left(\int_{\Omega} F\left(x, u_{m}, v_{m}\right) d x\right)^{\frac{p}{p^{*}}} \leq\left\|\left(u_{m}, v_{m}\right)\right\|_{E}^{p}$ we conclude, as in the proof of Lemma 2.1, that $N c_{0,0}=l \geq S_{F}^{\frac{N}{p}}$. Hence,

$$
c_{0,0}=\lim _{m \rightarrow \infty} I_{0,0}\left(u_{m}, v_{m}\right)=\lim _{m \rightarrow \infty}\left(\frac{1}{p}\left\|\left(u_{m}, v_{m}\right)\right\|_{E}^{p}-\frac{1}{p^{*}} \int_{\Omega} F\left(x, u_{m}, v_{m}\right) d x\right)=\frac{1}{N} l \geq \frac{1}{N} S_{F}^{\frac{N}{p}},
$$

and therefore $c_{0,0}=\frac{1}{N} S_{F}^{\frac{N}{p}}$.

We proceed now with the calculation of $\lim _{\lambda, \delta \rightarrow 0^{+}} c_{\lambda, \delta}$. Let $\left\{\lambda_{m}\right\},\left\{\delta_{m}\right\} \subset \mathbb{R}^{+}$such that $\lambda_{m}$, $\delta_{m} \rightarrow 0^{+}$. Since $\lambda_{m}, \delta_{m}$ are positive, we have that $\int_{\Omega}\left(\lambda_{m}|u|^{q}+\delta_{m}|v|^{q}\right) d x \geq 0$ whenever $(u, v)$ is nonnegative. Thus, for this kind of function, we have that $I_{\lambda_{m}, \delta_{m}}(u, v) \leq I_{0,0}(u, v)$.

It follows that

$$
\begin{aligned}
c_{\lambda_{m}, \delta_{m}} & =\inf _{(u, v) \neq(0,0)} \max _{t \geq 0} I_{\lambda_{m}, \delta_{m}}(t(u, v)) \\
& \leq \inf _{\substack{(u, v) \neq(0,0),(u, v) \geq 0}} \max _{t \geq 0} I_{\lambda_{m}, \delta_{m}}(t(u, v)) \\
& \leq \inf _{\substack{(u, v) \neq(0,0),(u, v) \geq 0}} \max _{t \geq 0} I_{0,0}(t(u, v))=c_{0,0},
\end{aligned}
$$

in the last equality, we have used the infimum $c_{0,0}$ which can be attained at a nonnegative solution. The above inequality implies that

$$
\limsup _{m \rightarrow \infty} c_{\lambda_{m}, \delta_{m}} \leq c_{0,0}
$$

On the other hand, it follows from Theorem 1.1 that there exists $\left\{\left(u_{m}, v_{m}\right)\right\} \subset E$ such that 


$$
I_{\lambda_{m}, \delta_{m}}\left(u_{m}, v_{m}\right)=c_{\lambda_{m}, \delta_{m}}, \quad I_{\lambda_{m}, \delta_{m}}^{\prime}\left(u_{m}, v_{m}\right) \rightarrow 0 .
$$

Since $c_{\lambda_{m}, \delta_{m}}$ is bounded, the same argument performed in the proof of Lemma 2.1 implies that $\left\{\left(u_{m}, v_{m}\right)\right\}$ is bounded in $E$. Since

$$
\lim _{m \rightarrow \infty} \int_{\Omega}\left(\lambda_{m}\left|u_{m}\right|^{q}+\delta_{m}\left|v_{m}\right|^{q}\right) d x=0 .
$$

Let $t_{m}>0$ be such that $t_{m}\left(u_{m}, v_{m}\right) \in \mathcal{N}_{0,0}$. Since $\left(u_{m}, v_{m}\right) \in \mathcal{N}_{\lambda_{m}, \delta_{m}}$, we have that

$$
\begin{aligned}
c_{0,0} & \leq I_{0,0}\left(t_{m}\left(u_{m}, v_{m}\right)\right) \\
& =I_{\lambda_{m}, \delta_{m}}\left(t_{m}\left(u_{m}, v_{m}\right)\right)+\frac{t_{m}^{q}}{q} \int_{\Omega}\left(\lambda_{m}\left|u_{m}\right|^{q}+\delta_{m}\left|v_{m}\right|^{q}\right) d x \\
& \leq I_{\lambda_{m}, \delta_{m}}\left(u_{m}, v_{m}\right)+\frac{t_{m}^{q}}{q} \int_{\Omega}\left(\lambda_{m}\left|u_{m}\right|^{q}+\delta_{m}\left|v_{m}\right|^{q}\right) d x \\
& =c_{\lambda_{m}, \delta_{m}}+\frac{t_{m}^{q}}{q} \int_{\Omega}\left(\lambda_{m}\left|u_{m}\right|^{q}+\delta_{m}\left|v_{m}\right|^{q}\right) d x .
\end{aligned}
$$

If $\left\{t_{m}\right\}$ is bounded, we can use the above estimate and (2.18) to get

$$
c_{0,0} \leq \liminf _{m \rightarrow \infty} c_{\lambda_{m}, \delta_{m}} .
$$

This and (2.17) we get

$$
c_{0,0} \leq \liminf _{m \rightarrow \infty} c_{\lambda_{m}, \delta_{m}} \leq \limsup _{m \rightarrow \infty} c_{\lambda_{m}, \delta_{m}} \leq c_{0,0},
$$

that is $c_{0,0}=\lim _{m \rightarrow \infty} \mathcal{C}_{\lambda_{m}, \delta_{m}}$.

It remains to check that $\left\{t_{m}\right\}$ is bounded. A straightforward calculation shows that

$$
t_{m}=\left(\frac{\left\|\left(u_{m}, v_{m}\right)\right\|_{E}^{p}}{\int_{\Omega} F\left(x, u_{m}, v_{m}\right) d x}\right)^{\frac{1}{p^{*}-p}} .
$$

Since $\left(u_{m}, v_{m}\right) \in \mathcal{N}_{\lambda_{m}, \delta_{m}}$, we obtain

$$
\left\|\left(u_{m}, v_{m}\right)\right\|_{E}^{p}=\int_{\Omega} F\left(x, u_{m}, v_{m}\right) d x+\int_{\Omega}\left(\lambda_{m}\left|u_{m}\right|^{q}+\delta_{m}\left|v_{m}\right|^{q}\right) d x \leq S_{F}^{-\frac{p^{*}}{p}}\left\|\left(u_{m}, v_{m}\right)\right\|_{E}^{p^{*}}+o_{m}(1) .
$$

Hence $\left\|\left(u_{m}, v_{m}\right)\right\|_{E}^{p} \geq C_{4}>0$, and therefore from the above expression it follows that $\int_{\Omega} F\left(x, u_{m}, v_{m}\right) d x \geq C_{5}>0$. Thus, the boundedness of $\left\{\left(u_{m}, v_{m}\right)\right\}$ and (2.19) imply that $\left\{t_{m}\right\}$ is bounded. This completes the proof.

\section{Some technical lemmas}

In this section, we denote by $\mathcal{M}(\Omega)$ the Banach space of finite Radon measures over $\Omega$ equipped with the norm $\|\sigma\|=\sup _{\substack{\varphi \in C_{0}(\Omega) \\|\varphi|_{\infty}=1}}|\sigma(\varphi)|$. A sequence $\left\{\sigma_{m}\right\} \subset \mathcal{M}(\Omega)$ is said to converge weakly to $\sigma \in \mathcal{M}(\Omega)$ provided $\sigma_{m}(\phi) \rightarrow \sigma(\phi)$ for all $\phi \in C_{0}(\Omega)$. By [18, Theorem 1.39], every bounded sequence $\left\{\sigma_{m}\right\} \subset \mathcal{M}(\Omega)$ contains a weakly convergent subsequence. 
The next lemma is a version of the second concentration-compactness lemma of Lions [21]. It is also inspired by [18, Lemma 1.40] and [[22], Lemma 2.4].

Lemma 3.1. Suppose that the sequence $\left\{\left(u_{m}, v_{m}\right)\right\} \subset D^{1, p}\left(\mathbb{R}^{N}\right) \times D^{1, p}\left(\mathbb{R}^{N}\right)$ satisfies

$$
\begin{aligned}
& \left(u_{m}, v_{m}\right) \rightarrow(u, v) \text { in } D^{1, p}\left(\mathbb{R}^{N}\right) \times D^{1, p}\left(\mathbb{R}^{N}\right), \\
& \left(u_{m}, v_{m}\right) \rightarrow(u, v) \text { a.e. } x \in \mathbb{R}^{N}, \\
& \left(\nabla u_{m}, \nabla v_{m}\right) \rightarrow(\nabla u, \nabla v) \text { a.e. } x \in \mathbb{R}^{N}, \\
& \left|\nabla\left(u_{m}-u\right)\right|^{p} \rightarrow \mu, \quad\left|\nabla\left(v_{m}-v\right)\right|^{p} \rightarrow \sigma \text { in } \mathcal{M}\left(\mathbb{R}^{N}\right), \\
& F\left(x, u_{m}-u, v_{m}-v\right) \rightarrow \text { vin } \mathcal{M}\left(\mathbb{R}^{N}\right),
\end{aligned}
$$

and define

$$
\left\{\begin{array}{l}
\mu_{\infty}=\lim _{R \rightarrow \infty} \limsup _{m \rightarrow \infty} \int_{|x|>R}\left|\nabla u_{m}\right|^{p} d x \\
\sigma_{\infty}=\lim _{R \rightarrow \infty} \limsup _{m \rightarrow \infty} \int_{|x|>R}\left|\nabla v_{m}\right|^{p} d x, \\
v_{\infty}=\lim _{R \rightarrow \infty} \limsup _{m \rightarrow \infty} \int_{|x|>R} F\left(x, u_{m}, v_{m}\right) d x
\end{array}\right.
$$

then it follows that

$$
\begin{aligned}
& \limsup _{m \rightarrow \infty} \int_{\mathbb{R}^{N}}\left|\nabla u_{m}\right|^{p} d x=\|\mu\|+\mu_{\infty}+\int_{\mathbb{R}^{N}}|\nabla u|^{p} d x, \\
& \limsup _{m \rightarrow \infty} \int_{\mathbb{R}^{N}}\left|\nabla v_{m}\right|^{p} d x=\|\sigma\|+\sigma_{\infty^{+}} \int_{\mathbb{R}^{N}}|\nabla u|^{p} d x, \\
& \limsup _{m \rightarrow \infty} \int_{\mathbb{R}^{N}} F\left(x, u_{m}, v_{m}\right) d x=\|v\|+v_{\infty}+\int_{\mathbb{R}^{N}} F(x, u, v) d x, \\
& \|v\| \frac{p}{p^{*}} \leq S_{F}^{-1}(\|\mu\|+\|\sigma\|), \quad \frac{p}{p_{\infty}^{*}} \leq S_{F}^{-1}\left(\mu_{\infty}+\sigma_{\infty}\right) .
\end{aligned}
$$

Moreover, if $(u, v)=(0,0)$ and $\frac{p \|}{p^{*}}=S_{F}^{-1}(\|\mu\|+\|\sigma\|)$, then the measures $\mu, v$, and $\sigma$ are concentrated at a single point, respectively.

Proof. We first recall that, in view of the definition of $S_{F}$, for each nonnegative function $\varphi \in C_{0}^{\infty}\left(\mathbb{R}^{N}\right)$ we have

$$
\left(\int_{\mathbb{R}^{N}}|\varphi|^{p^{*}} F\left(x, u_{m}, v_{m}\right) d x\right)^{\frac{p}{p^{*}}}=\left(\int_{\mathbb{R}^{N}} F\left(x, \varphi u_{m}, \varphi v_{m}\right) d x\right)^{\frac{p}{p^{*}}} \leq S_{F}^{-1}\left\|\left(\varphi u_{m}, \varphi v_{m}\right)\right\|_{E}^{p} .
$$

Moreover, arguing as [8, Lemma 5], we have that

$$
\int_{\mathbb{R}^{N}} \varphi F\left(x, u_{m}-u, v_{m}-v\right) d x=\int_{\mathbb{R}^{N}} \varphi F\left(x, u_{m}, v_{m}\right) d x-\int_{\mathbb{R}^{N}} \varphi F(x, u, v) d x+o_{m}(1) .
$$

Since $F$ is $p^{*}$-homogeneous, we can use the two above expressions and argue along the same line of the proof of Lemma 1.40 in [18] to conclude that (3.2)-(3.5) hold. If 
$(u, v)=(0,0)$ and ${ }_{\|v\|} \frac{p}{p^{*}}=S_{F}^{-1}(\|\mu\|+\|\sigma\|)$, the same argument of step 3 of the proof of Lemma 1.40 in [18] implies that the measures $\mu, v$ and $\sigma$ are concentrated at a single point, respectively.

Remark 3.1. We notice that the last conclusion of the above result holds even if $(u, v)$ $\nabla(0,0)$. Indeed, in this case we can define $\left(\tilde{u}_{m}, \tilde{v}_{m}\right)=\left(u_{m}-u, v_{m}-v\right)$ and notice that

$$
\begin{aligned}
& \left(\tilde{u}_{m}, \tilde{v}_{m}\right) \rightarrow(\tilde{u}, \tilde{v}) \text { in } D^{1, p}\left(\mathbb{R}^{N}\right) \times D^{1, p}\left(\mathbb{R}^{N}\right), \\
& \left(\tilde{u}_{m}, \tilde{v}_{m}\right) \rightarrow(0,0) \text { a.e. } x \in \mathbb{R}^{N}, \\
& \left(\nabla \tilde{u}_{m}, \nabla \tilde{v}_{m}\right) \rightarrow(\nabla \tilde{u}, \nabla \tilde{v}) \text { a.e. } x \in \mathbb{R}^{N}, \\
& \left|\nabla\left(\tilde{u}_{m}-\tilde{u}\right)\right|^{p} \rightarrow \tilde{\mu}, \quad\left|\nabla\left(\tilde{v}_{m}-\tilde{v}\right)\right|^{p} \rightarrow \tilde{\sigma} \text { in } \mathcal{M}\left(\mathbb{R}^{N}\right), \\
& F\left(x, \tilde{u}_{m}-\tilde{u}, \tilde{v}_{m}-\tilde{v}\right) \rightarrow \tilde{v} \text { in } \mathcal{M}\left(\mathbb{R}^{N}\right),
\end{aligned}
$$

Since $\left(\tilde{u}_{m}-\tilde{u}, \tilde{v}_{m}-\tilde{v}\right)=\left(u_{m}-u, v_{m}-v\right)$ and therefore $\tilde{\mu}=\mu, \tilde{\sigma}=\sigma$, and $\tilde{v}=v$, where $\mu, \sigma$, and $v$ are the same as those in Lemma 3.1. Thus, if $\frac{\|v\|}{\frac{p}{p^{*}}}=S_{F}^{-1}(\|\mu\|+\|\sigma\|)$ we also have that ${ }_{\|\tilde{v}\|} \frac{p}{p^{*}}=S_{F}^{-1}(\|\tilde{\mu}\|+\|\tilde{\sigma}\|)$ and the result follows from the last part of Lemma 3.1.

Now, we introduce the following Lemma.

Lemma 3.2. Suppose $\left\{\left(u_{m}, v_{m}\right)\right\} \subset E$ such that $\int_{\Omega} F\left(x, u_{m}, v_{m}\right) d x=1$ and $\lim _{m \rightarrow \infty}\left\|\left(u_{m}, v_{m}\right)\right\|_{E}^{p}=S_{F}$. Then there exist $\left\{r_{m}\right\} \subset(0,+\infty)$ and $\left\{y_{m}\right\} \subset \mathbb{R}^{N}$ such that

$$
\left(\omega_{m}^{1}(x), \omega_{m}^{2}(x)\right)=r_{m}^{\frac{N-p}{p}}\left(u_{m}\left(r_{m} x+y_{m}\right), v_{m}\left(r_{m} x+y_{m}\right)\right)
$$

contains a convergent subsequence denoted again by $\left\{\left(\omega_{m}^{1}(x), \omega_{m}^{2}(x)\right)\right\}$ such that $\left(\omega_{m}^{1}(x), \omega_{m}^{2}(x)\right) \rightarrow\left(\omega_{1}, \omega_{2}\right)$ in $D^{1, p}\left(\mathbb{R}^{N}\right) \times D^{1, p}\left(\mathbb{R}^{N}\right)$. Moreover, as $m \rightarrow \infty$, we have $r_{m} \rightarrow$ 0 and $y_{m} \rightarrow y \in \bar{\Omega}$.

Proof. For each $r>0$, we consider the Lévy concentration functions

$$
H_{m}(r)=\sup _{\gamma \in \mathbb{R}^{N}} \int_{B_{r}(y)} F\left(x, u_{m}, v_{m}\right) d x .
$$

Since for every $m$,

$$
\lim _{r \rightarrow 0^{+}} H_{m}(r)=0, \quad \lim _{r \rightarrow \infty} H_{m}(r)=1,
$$

there exist $r_{m}>0$ and a sequence $\left\{y_{m}^{k}\right\} \subset \mathbb{R}^{N}$ satisfying

$$
\frac{1}{2}=H_{m}\left(r_{m}\right)=\lim _{k \rightarrow \infty} \int_{B_{r m}\left(y_{m}^{k}\right)} F\left(x, u_{m}, v_{m}\right) d x
$$

Recalling that $\lim _{|y| \rightarrow \infty} \int_{B_{r_{m}}(y)} F\left(x, u_{m}, v_{m}\right) d x=0$, we conclude that $\left\{\gamma_{m}^{k}\right\}$ is bounded.

Hence, up to a subsequence, $\lim _{k \rightarrow \infty} \gamma_{m}^{k}=\gamma_{m} \in \mathbb{R}^{N}$ and we obtain 


$$
\frac{1}{2}=\int_{B_{r m}\left(y_{m}\right)} F\left(x, u_{m}, v_{m}\right) d x
$$

We shall prove that the above sequences $\left\{r_{m}\right\}$ and $\left\{y_{m}\right\}$ satisfy the statements of the lemma. First notice that

$$
\frac{1}{2}=\int_{B_{r_{m}}\left(y_{m}\right)} F\left(x, u_{m}, v_{m}\right) d x=\int_{B_{1}(0)} F\left(x, \omega_{m}^{1}, \omega_{m}^{2}\right) d x=\sup _{y \in \mathbb{R}^{N}} \int_{B_{1}(\gamma)} F\left(x, \omega_{m}^{1}, \omega_{m}^{2}\right) d x .
$$

By (3.6), a straightforward calculation provides

$$
\lim _{m \rightarrow \infty}\left\|\left(\omega_{m}^{1}, \omega_{m}^{2}\right)\right\|_{E}^{p}=\lim _{m \rightarrow \infty}\left\|\left(u_{m}, v_{m}\right)\right\|_{E}^{p}=S_{F} \int_{\mathbb{R}^{N}} F\left(x, \omega_{m}^{1}, \omega_{m}^{2}\right) d x=1 .
$$

Hence, we can apply Lemma 3.1 to obtain $\left(\omega_{1}, \omega_{2}\right) \in D^{1, p}\left(\mathbb{R}^{N}\right) \times D^{1, p}\left(\mathbb{R}^{N}\right)$ satisfying

$$
\begin{aligned}
& S_{F}=\|\mu\|+\mu_{\infty}+\|\sigma\|+\sigma_{\infty}+\left\|\left(\omega_{1}, \omega_{2}\right)\right\|_{E^{\prime}}^{p} \quad 1=\|v\|+v_{\infty}+\int_{\mathbb{R}^{N}} F\left(x, \omega_{1}, \omega_{2}\right) d x \\
& \|v\| \frac{p}{p^{*}} \leq S_{F}^{-1}(\|\mu\|+\|\sigma\|), \quad \frac{p}{v_{\infty}^{*}} \leq S_{F}^{-1}\left(\mu_{\infty}+\sigma_{\infty}\right) .
\end{aligned}
$$

The second equality in (3.8) implies that $\|v\|, v_{\infty}, \int_{\mathbb{R}^{N}} F\left(x, \omega_{1}, \omega_{2}\right) d x \in[0,1]$. If one of these values belongs to the open interval $(0,1)$, we can use (3.8), $\frac{p}{p^{*}}<1,\left(\int_{\mathbb{R}^{N}} F\left(x, \omega_{1}, \omega_{2}\right) d x\right)^{\frac{p}{p^{*}}} \leq S_{F}^{-1}\left\|\left(\omega_{1}, \omega_{2}\right)\right\|_{E}^{p}$ and (3.9) to get

$$
\begin{aligned}
S_{F} & =S_{F}\left(\|v\|+v_{\infty}+\int_{\mathbb{R}^{N}} F\left(x, \omega_{1}, \omega_{2}\right) d x\right) \\
& <S_{F}\left(\|v\|^{\frac{p}{p^{*}}}+v_{\infty}^{\frac{p}{p^{*}}}+\left(\int_{\mathbb{R}^{N}} F\left(x, \omega_{1}, \omega_{2}\right) d x\right)^{\frac{p}{p^{*}}}\right) \\
& \leq S_{F},
\end{aligned}
$$

which is a contradiction. Thus $\|v\|, v_{\infty}, \int_{\mathbb{R}^{N}} F\left(x, \omega_{1}, \omega_{2}\right) d x \in\{0,1\}$. Actually, it follows from (3.7) that $\int_{|x|>R} F\left(x, \omega_{m}^{1}, \omega_{m}^{2}\right) d x \leq \frac{1}{2}$ for any $R>1$. Thus, we conclude that $v_{\infty}=0$.

Let us prove that $\|v\|=0$. Arguing by contradiction, then $\|v\|=1$. It follows from the first equality in (3.8) that $S_{F} \geq\|\mu\|+\|\sigma\|$. On the other hand, the first inequality in (3.9) provides $\|\mu\|+\|\sigma\| \geq S_{F}$. Hence, we conclude that $\|\mu\|+\|\sigma\|=S_{F}$. Since we suppose that $\|v\|=1$ we obtain $\|v\| \frac{p}{p^{*}}=S_{F}^{-1}(\|\mu\|+\|\sigma\|)$. It follows from Remark 3.1 that $v=\delta_{x_{0}}$ for some $x_{0} \in \mathbb{R}^{N}$. Thus, from (3.7), we get

$$
\frac{1}{2} \geq \lim _{m \rightarrow \infty} \int_{B_{1}\left(x_{0}\right)} F\left(x, \omega_{m}^{1}, \omega_{m}^{2}\right) d x=\int_{B_{1}\left(x_{0}\right)} d v=\|v\|=1 .
$$

This contradiction proves that $\|\nu\|=0$. 
Since $\|v\|=v_{\infty}=0$, we have that $\int_{\mathbb{R}^{N}} F\left(x, \omega_{1}, \omega_{2}\right) d x=1$. This and (3.8) provide

$$
\lim _{m \rightarrow \infty}\left\|\left(\omega_{m^{\prime}}^{1} \omega_{m}^{2}\right)\right\|_{E}^{p}=S_{F} \geq\left\|\left(\omega_{1}, \omega_{2}\right)\right\|_{E}^{p} \geq S_{F}\left(\int_{\mathbb{R}^{N}} F\left(x, \omega_{1}, \omega_{2}\right) d x\right)^{\frac{p}{p^{*}}}=S_{F} .
$$

So, $\left\|\left(\omega_{1}, \omega_{2}\right)\right\|_{E}^{p}=S_{F}$ and therefore $\left(\omega_{m}^{1}, \omega_{m}^{2}\right) \rightarrow\left(\omega_{1}, \omega_{2}\right) \not \equiv(0,0)$ strongly in $D^{1, p}\left(\mathbb{R}^{N}\right)$ $\times D^{1, p}\left(\mathbb{R}^{N}\right)$ and $\left(\omega_{m}^{1}(x), \omega_{m}^{2}(x)\right) \rightarrow\left(\omega_{1}(x), \omega_{2}(x)\right)$ for a.e. $x \in \mathbb{R}^{N}$. In order to conclude the proof we notice that

$$
\left\|\left(\omega_{m^{\prime}}^{1} \omega_{m}^{2}\right)\right\|_{L^{p}\left(\mathbb{R}^{N}\right) \times L^{p}\left(\mathbb{R}^{N}\right)}=\frac{1}{r_{m}^{p}}\left\|\left(u_{m}, v_{m}\right)\right\|_{L^{p}(\Omega) \times L^{p}(\Omega) .}
$$

Since $\left\{\left(u_{m}, v_{m}\right)\right\}$ is bounded and $\left(\omega_{1}, \omega_{2}\right) \otimes(0,0)$, we infer from the above equality that, up to a subsequence, $r_{m} \rightarrow r_{0} \geq 0$. If $\left|y_{m}\right| \rightarrow \infty$, for each fixed $x \in \mathbb{R}^{N}$, we have that there exists $m_{x} \in N$ such that $r_{m} x+y_{m} \notin \Omega$ for $m \geq m_{x}$. For such values of $m$ we have that $\left(\omega_{m}^{1}(x), \omega_{m}^{2}(x)\right)=(0,0)$. Taking the limit and recalling that $x \in \mathbb{R}$ is arbitrary, we conclude that $\left(\omega_{1}, \omega_{2}\right)=(0,0)$, which is a contradiction. So, along a subsequence, $y_{m} \rightarrow y \in \mathbb{R}^{N}$.

We claim that $r_{0}=0$. Indeed, suppose by contradiction that $r_{0}>0$. Then, as $m$ becomes large, the set $\Omega_{m}=\left(\Omega-y_{m}\right) / r_{m}$ approaches $\Omega_{0}=(\Omega-y) / r_{0} \neq \mathbb{R}^{N}$. This implies that $\omega_{1}, \omega_{2}$ has compact support in $\mathbb{R}^{N}$. On the other hand, since $\left(\omega_{1}, \omega_{2}\right)$ achieves the infimum in (2.3) and $F$ is homogeneous, we can use the Lagrange Multiplier Theorem to conclude that $\left(\omega_{1}, \omega_{2}\right)$ satisfies

$$
-\Delta_{p} u=\theta \frac{\partial F(x, u, v)}{\partial u},-\Delta_{p} v=\theta \frac{\partial F(x, u, v)}{\partial u}, x \in \mathbb{R}^{N},
$$

for $\theta=\frac{S_{F}}{p^{*}}>0$. It follows from $\left(F_{2}\right)$ and the maximum principle that at least one of the functions $\omega_{1}, \omega_{2}$ is positive in $\mathbb{R}^{N}$. But this contradicts supp $\left(\omega_{1}, \omega_{2}\right) \subset \Omega_{0}$. Hence, we conclude that $r_{0}=0$. Finally, if $y \notin \bar{\Omega}$ we obtain $r_{m} x+y_{m} \notin \Omega$ for large values of $m$, and therefore we should have $\left(\omega_{1}, \omega_{2}\right) \equiv(0,0)$ again. Thus, $y \in \bar{\Omega}$ and the proof is completed.

Up to translations, we may assume that $0 \in \Omega$, since $\Omega$ is a smooth bounded domain of $\mathbb{R}^{N}$, we can choose $r>0$ small enough such that $B_{r}=B_{r}(0)=\left\{x \in \mathbb{R}^{N}: d(x, 0)<r\right\} \subset$ $\Omega$ and the sets

$$
\Omega_{r}^{+}=\left\{x \in \mathbb{R}^{N}: \operatorname{dist}(x, \Omega)<r\right\}, \quad \Omega_{r}^{-}=\left\{x \in \mathbb{R}^{N}: \operatorname{dist}(x, \partial \Omega)>r\right\},
$$

are homotopically equivalent to $\Omega$. Let

$$
W_{0, \text { rad }}^{1, p}\left(B_{r}\right)=\left\{u \in W_{0}^{1, p}\left(B_{r}\right): u \text { is radial }\right\}
$$

and

$$
E_{\mathrm{rad}}\left(B_{r}\right)=W_{0, \mathrm{rad}}^{1, p}\left(B_{r}\right) \times W_{0, \mathrm{rad}}^{1, p}\left(B_{r}\right) .
$$


We define the functional

$$
I_{B_{r}}(u, v)=\frac{1}{p} \int_{B_{r}}\left(|\nabla u|^{p}+|\nabla v|^{p}\right) d x-\frac{1}{p^{*}} \int_{B_{r}} F(x, u, v) d x-\frac{1}{q} \int_{B_{r}}\left(\lambda|u|^{q}+\delta|v|^{q}\right) d x,(u, v) \in E_{\mathrm{rad}}\left(B_{r}\right),
$$

and set

$$
m_{\lambda, \delta}=\inf _{(u, v) \in \mathcal{N}_{\lambda, \delta}^{B_{r}}} I_{B_{r}}(u, v),
$$

where

$$
\mathcal{N}_{\lambda, \delta}^{B_{r}}:=\left\{(u, v) \in E_{\text {rad }}\left(B_{r}\right) \backslash\{(0,0)\}:\left\langle I_{B_{r}}^{\prime}(u, v),(u, v)\right\rangle=0\right\} .
$$

Clearly, $m_{\lambda, \delta}$ is nonincreasing in $\lambda, \delta$. Note that $m_{\lambda, \delta}>0$ for all $\lambda, \delta>0$.

Arguing as in the proof of Lemma 2.3 and Theorem 1.1, we obtain the following result.

Lemma 3.3. Suppose $F$ satisfies $\left(F_{0}\right)-\left(F_{2}\right)$, then the infimum $m_{\lambda, \delta}$ is attained by a nonneg-ative radial function $\left(u_{\lambda, \delta}, v_{\lambda, \delta}\right) \in E_{\text {rad }}$ whenever $2 \leq p<q<p^{*}$ and $\lambda, \delta>0$, or $q=p$ and $\lambda, \delta \in\left(0, \Lambda_{1, \mathrm{rad}}\right)$, where $\Lambda_{1, \mathrm{rad}}>0$ is the first eigenvalue of the operator $\left(-\Delta_{p}, W_{0, \text { rad }}^{1, p}\left(B_{r}\right)\right)$. Moreover,

$$
m_{\lambda, \delta}<\frac{1}{N} S_{F}^{\frac{N}{p}}, \lim _{\lambda, \delta \rightarrow 0^{+}} m_{\lambda, \delta}=\frac{1}{N} S_{F}^{\frac{N}{p}} .
$$

We introduce the barycenter map $\beta: \mathcal{N}_{\lambda, \delta} \rightarrow \mathbb{R}^{N}$ as follows

$$
\beta(u, v)=S_{F}^{-\frac{N}{p}} \int_{\Omega} F(x, u, v) x d x .
$$

This map has the following property.

Lemma 3.4. If $N \geq p^{2}, 2 \leq p \leq q<p^{*}$ and F satisfies $\left(F_{0}\right)-\left(F_{2}\right)$, then there exists $\lambda^{*}>0$ such that $\beta(u, v) \in \Omega_{r}^{+}$whenever $(u, v) \in \mathcal{N}_{\lambda, \delta}, \lambda, \delta \in\left(0, \lambda^{*}\right)$ and $I_{\lambda, \delta}(u, v) \leq m_{\lambda, \delta}$.

Proof. By way of contradiction, we suppose that there exist $\left\{\lambda_{m}\right\},\left\{\delta_{m}\right\} \subset \mathbb{R}^{+}$and $\left\{\left(u_{m}, v_{m}\right)\right\} \subset \mathcal{N}_{\lambda_{m}, \delta_{m}}$ such that $\lambda_{m}, \delta_{m} \rightarrow 0^{+}$as $m \rightarrow \infty, I_{\lambda_{m}, \delta_{m}}\left(u_{m}, v_{m}\right) \leq m_{\lambda_{m}, \delta_{m}}$ but $\beta\left(u_{m}, v_{m}\right) \notin \Omega_{r}^{+}$.

From $\left\{\left(u_{m}, v_{m}\right)\right\} \subset \mathcal{N}_{\lambda_{m}, \delta_{m}}$ and $I_{\lambda_{m}, \delta_{m}}\left(u_{m}, v_{m}\right) \leq m_{\lambda_{m}, \delta_{m}}$ we have that $\left\{\left(u_{m}, v_{m}\right)\right\}$ is bounded in $E$. Moreover,

$$
0=\left\langle I_{\lambda_{m}, \delta_{m}}^{\prime}\left(u_{m}, v_{m}\right),\left(u_{m}, v_{m}\right)\right\rangle=\left\|\left(u_{m}, v_{m}\right)\right\|_{E}^{p}-\int_{\Omega} F\left(x, u_{m}, v_{m}\right) d x-\int_{\Omega}\left(\lambda_{m}\left|u_{m}\right|^{q}+\delta_{m}\left|v_{m}\right|^{q}\right) d x .
$$

Since $\lambda_{m}, \delta_{m} \rightarrow 0^{+}$, we can use the boundedness of $\left\{\left(u_{m}, v_{m}\right)\right\}$ to get

$$
0 \leq \int_{\Omega}\left(\lambda_{m}\left|u_{m}\right|^{q}+\delta_{m}\left|v_{m}\right|^{q}\right) d x \rightarrow 0,
$$

from which it follows that

$$
\lim _{m \rightarrow \infty}\left\|\left(u_{m}, v_{m}\right)\right\|_{E}^{p}=\lim _{m \rightarrow \infty} \int_{\Omega} F\left(x, u_{m}, v_{m}\right) d x=k \geq 0
$$


Notice that

$$
\begin{aligned}
c_{\lambda_{m}, \delta_{m}} & \leq I_{\lambda_{m}, \delta_{m}}\left(u_{m}, v_{m}\right) \\
& =\frac{1}{p}\left\|\left(u_{m}, v_{m}\right)\right\|_{E}^{p}-\frac{1}{p^{*}} \int_{\Omega} F\left(x, u_{m}, v_{m}\right) d x-\frac{1}{q} \int_{\Omega}\left(\lambda_{m}\left|u_{m}\right|^{q}+\delta_{m}\left|v_{m}\right|^{q}\right) d x \\
& \leq m_{\lambda_{m}, \delta_{m}} .
\end{aligned}
$$

Recalling that $c_{\lambda_{m}, \delta_{m}}$ and $m_{\lambda_{m}}, \delta_{m}$ both converge to $\frac{1}{N} S_{F}^{\frac{N}{p}}$, we can use the above expression and $\int_{\Omega}\left(\lambda_{m}\left|u_{m}\right|^{q}+\delta_{m}\left|v_{m}\right|^{q}\right) d x \rightarrow 0$ again to conclude that $k=S_{F}^{\frac{N}{p}}$, that is,

$$
\lim _{m \rightarrow \infty}\left\|\left(u_{m}, v_{m}\right)\right\|_{E}^{p}=S_{F}^{\frac{N}{p}}=\lim _{m \rightarrow \infty} \int_{\Omega} F\left(x, u_{m}, v_{m}\right) d x .
$$

Let $t_{m}=\left(\int_{\Omega} F\left(x, u_{m}, v_{m}\right) d x\right)^{-\frac{1}{p^{*}}}>0$ and notice that $t_{m}\left(u_{m}, v_{m}\right)$ satisfies the hypotheses of Lemma 3.2. Using Lemma 3.2, there exist sequences $\left\{r_{m}\right\} \subset(0,+\infty)$ and $\left\{y_{m}\right\} \subset$ $\mathbb{R}^{N}$ satisfying $r_{m} \rightarrow 0, y_{m} \rightarrow y \in \bar{\Omega}$ we have that $\left(\omega_{m}^{1}, \omega_{m}^{2}\right) \rightarrow\left(\omega_{1}, \omega_{2}\right)$ in $D^{1, p}\left(\mathbb{R}^{N}\right) \times D^{1,}$ $p\left(\mathbb{R}^{N}\right)$.

The definition of $\beta(u, v)$, (3.10), the strong convergence of $\left\{\left(\omega_{m}^{1}, \omega_{m}^{2}\right)\right\}$ and Lebesgue's theorem provide

$$
\begin{aligned}
\beta\left(u_{m}, v_{m}\right) & =t_{m}^{-p^{*}} S_{F}^{-\frac{N}{p}} \int_{\Omega} F\left(x, t_{m}\left(u_{m}, v_{m}\right)\right) x d x \\
& =\left(1+o_{m}(1)\right) \int_{\Omega} F\left(x, t_{m}\left(u_{m}, v_{m}\right)\right) x d x \\
& =\left(1+o_{m}(1)\right) \int_{\Omega} F\left(x, \omega_{m}^{1}, \omega_{m}^{2}\right)\left(r_{m} x+y_{m}\right) d x \\
& =\left(1+o_{m}(1)\right)\left(\int_{\Omega} F\left(x, \omega_{1}, \omega_{2}\right) \bar{y} d x+o_{m}(1)\right) .
\end{aligned}
$$

Since $\bar{y} \in \bar{\Omega}$ and $\int_{\Omega} F\left(x, \omega_{1}, \omega_{2}\right) d x=1$, the above expression implies that

$$
\lim _{m \rightarrow \infty} \operatorname{dist}\left(\beta\left(u_{m}, v_{m}\right), \bar{\Omega}\right)=0,
$$

which contradicts $\beta\left(u_{m}, v_{m}\right) \notin \Omega_{r}^{+}$.

According to Lemma 3.3, for each $\lambda, \delta>0$ small the infimum $m_{\lambda, \delta}$ is attained by a nonnegative radial function $\sigma_{\lambda, \delta}=\left(u_{\lambda, \delta}, v_{\lambda, \delta}\right) \in \mathcal{N}_{\lambda, \delta}^{B_{r}}$. We consider

$$
I_{\lambda, \delta}^{m_{\lambda, \delta}}=\left\{(u, v) \in E: I_{\lambda, \delta}(u, v) \leq m_{\lambda, \delta}\right\}
$$

and define the function $\gamma: \Omega_{r}^{-} \rightarrow I_{\lambda, \delta}^{m, \delta}$ by setting, for each $\gamma \in \Omega_{r}^{-}$,

$$
\gamma(\gamma)= \begin{cases}\sigma_{\lambda, \delta}(x-y), & \text { if } x \in B_{r}(y), \\ 0, & \text { otherwise. }\end{cases}
$$


A change of variables and straightforward calculations show that the map $\gamma$ is well defined. Since $\sigma_{\lambda, \delta}$ is radial, we have that $\int_{B_{r}} F\left(x, u_{\lambda, \delta}, v_{\lambda, \delta}\right) x d x=0$. Hence, for each $y \in \Omega_{r}^{-}$, we obtain

$$
\begin{aligned}
(\beta \circ \gamma)(\gamma) & =S_{F}^{-\frac{N}{p}} \int_{\Omega} F\left(x-y, u_{\lambda, \delta}(x-y), v_{\lambda, \delta}(x-y)\right) x d x \\
& =S_{F}^{-\frac{N}{p}} \int_{\Omega} F\left(t, u_{\lambda, \delta}(t), v_{\lambda, \delta}(t)\right)(t+y) d t \\
& =S_{F}^{-\frac{N}{p}} \int_{\Omega} F\left(t, u_{\lambda, \delta}(t), v_{\lambda, \delta}(t)\right) y d t \\
& =\gamma \alpha_{\lambda, \delta}
\end{aligned}
$$

where $\alpha_{\lambda, \delta}=S_{F}^{-\frac{N}{p}} \int_{\Omega} F\left(t, u_{\lambda, \delta}(t), v_{\lambda, \delta}(t)\right) d t$.

Along the way of proving Lemma 3.4 we can check easily the following

Lemma 3.5. If $\lambda, \delta \rightarrow 0^{+}, \alpha_{\lambda, \delta} \rightarrow 1$.

Proof. By Lemma 3.3, we have

$$
m_{\lambda, \delta}=\frac{1}{p} \int_{B_{r}}\left(\left|\nabla u_{\lambda, \delta}\right|^{p}+\left|\nabla v_{\lambda, \delta}\right|^{p}\right) d x-\frac{1}{p^{*}} \int_{B_{r}} F\left(x, u_{\lambda, \delta}, v_{\lambda, \delta}\right) d x-\frac{1}{q} \int_{B_{r}}\left(\lambda\left|u_{\lambda, \delta}\right|^{q}+\delta\left|v_{\lambda, \delta}\right|^{q}\right) d x<\frac{1}{N} S_{F}^{\frac{N}{p}} .
$$

As before $\int_{B_{r}}\left(\lambda\left|u_{\lambda, \delta}\right|^{q}+\delta\left|v_{\lambda, \delta}\right|^{q}\right) d x \rightarrow 0$. Thus, $I_{B_{r}}^{\prime}\left(u_{\lambda, \delta}, v_{\lambda, \delta}\right)=0$, the above expression and the same arguments used in the proof of Lemma 3.3 imply that

$$
\int_{\Omega} F\left(x, u_{\lambda, \delta}, v_{\lambda, \delta}\right) d x \rightarrow S_{F}^{\frac{N}{p}} .
$$

The above equality and the definition of $\alpha_{\lambda, \delta}$ imply that $\alpha_{\lambda, \delta} \rightarrow 1$. The lemma is proved.

Next we define $H_{\lambda, \delta}:[0,1] \times\left(\mathcal{N}_{\lambda, \delta} \cap I_{\lambda, \delta}^{m_{\lambda, \delta}}\right) \rightarrow \mathbb{R}^{N}$ by

$$
H_{\lambda, \delta}(t,(u, v))=\left(t+\frac{1-t}{\alpha_{\lambda, \delta}}\right) \beta(u, v) .
$$

We have the following

Lemma 3.6. if $F$ satisfies $\left(F_{0}\right)-\left(F_{2}\right)$, then there exists $\lambda^{* * *}>0$ such that

$$
H_{\lambda, \delta}\left([0,1] \times\left(\mathcal{N}_{\lambda, \delta} \cap I_{\lambda, \delta}^{m_{\lambda, \delta}}\right)\right) \subset \Omega_{r}^{+},
$$

for all $\lambda, \delta \in\left(0, \lambda^{* * *}\right)$.

Proof. Arguing by contradiction, we suppose that there exist sequences $\left\{\lambda_{m}\right\},\left\{\delta_{m}\right\} \subset \mathbb{R}$ ${ }^{+}$and $t_{m} \in[0,1],\left(u_{m}, v_{m}\right) \in\left(\mathcal{N}_{\lambda, \delta} \cap I_{\lambda, \delta}^{m_{\lambda, \delta}}\right)$ such that $\lambda_{m}, \delta_{m} \rightarrow 0^{+}$, as $m \rightarrow \infty$, and $H_{\lambda_{m}, \delta_{m}}\left(t_{m},\left(u_{m}, v_{m}\right)\right) \notin \Omega_{r}^{+}$for all $m$. Up to a subsequence $t_{m} \rightarrow t_{0} \in[0,1]$. Moreover, the compactness of $\bar{\Omega}$ and Lemma 3.4 imply that, up to a subsequence, $\beta\left(u_{m}, v_{m}\right) \rightarrow y \in \bar{\Omega}$. From Lemma $3.5 \alpha_{\lambda_{m}, \delta_{m}} \rightarrow 1$. So, we can use the definition of $H_{\lambda, \delta}$ to conclude that $H_{\lambda_{m}, \delta_{m}}\left(t_{m},\left(u_{m}, v_{m}\right)\right) \rightarrow y \in \bar{\Omega}$, which is a contradiction. The lemma is proved. 


\section{Proof of Theorem $\mathbf{1 . 2}$}

We begin with the following lemma.

Lemma 4.1. If $(u, v)$ is a critical point of $I_{\lambda, \delta}$ on $\mathcal{N}_{\lambda, \delta}$, then it is a critical point of $I_{\lambda, \delta}$ in $E$.

Proof. The proof is almost the same as that [4, Lemma 4.1] and is omitted here.

Lemma 4.2. Suppose F satisfies $\left(F_{0}\right)-\left(F_{2}\right)$, then any sequence $\left\{\left(u_{m}, v_{m}\right)\right\} \subset \mathcal{N}_{\lambda, \delta}$ such that $I_{\lambda, \delta}\left(u_{m}, v_{m}\right) \rightarrow c<\frac{1}{N} S_{F}^{\frac{N}{p}}$ and $I_{\lambda, \delta}^{\prime}\left(u_{m}, v_{m}\right) \rightarrow 0$ contains a convergent subsequence for $\lambda, \delta>0$ if $q>p$ and $\lambda, \delta \in\left(0, \lambda^{*}\right)$ if $q=p$ for some small $\lambda^{*}>0$.

Proof. By hypothesis there exists a sequence $\theta_{m} \in \mathbb{R}$ such that $\left\|I_{\lambda, \delta}^{\prime}\left(u_{m}, v_{m}\right)-\theta_{m} J_{\lambda, \delta}^{\prime}\left(u_{m}, v_{m}\right)\right\|_{E} \rightarrow 0$ as $m \rightarrow \infty$, where $J_{\lambda, \delta}(u, v)=\left\langle I_{\lambda, \delta}^{\prime}(u, v),(u, v)\right\rangle$. Thus

$$
I_{\lambda, \delta}^{\prime}\left(u_{m}, v_{m}\right)=\theta_{m} J_{\lambda, \delta}^{\prime}\left(u_{m}, v_{m}\right)+o_{m}(1)
$$

Recall that

$$
\left\langle J_{\lambda, \delta}^{\prime}\left(u_{m}, v_{m}\right),\left(u_{m}, v_{m}\right)\right\rangle \leq 0 \text { for all }\left(u_{m}, v_{m}\right) \in \mathcal{N}_{\lambda, \delta} .
$$

If $\left\langle J_{\lambda, \delta}^{\prime}\left(u_{m}, v_{m}\right),\left(u_{m}, v_{m}\right)\right\rangle \rightarrow 0$, we have

$$
\int_{\Omega}\left(\lambda\left|u_{m}\right|^{q}+\delta\left|v_{m}\right|^{q}\right) d x \rightarrow 0, \quad \int_{\Omega} F\left(x, u_{m}, v_{m}\right) d x \rightarrow 0 .
$$

Consequently $\left\|\left(u_{m}, v_{m}\right)\right\|_{E} \rightarrow 0$.

On the other hand, if $\left(u_{m}, v_{m}\right) \subset \mathcal{N}_{\lambda, \delta}$ it follows that

$$
1 \leq C\left(\lambda\left\|\left(u_{m}, v_{m}\right)\right\|_{E}^{q-p}+\delta\left\|\left(u_{m}, v_{m}\right)\right\|_{E}^{q-p}+\left\|\left(u_{m}, v_{m}\right)\right\|_{E}^{p^{*}-p}\right)
$$

for some $C>0$. Hence we arrive at a contradiction if $\lambda, \delta>0$ and $q>p$ or $\lambda, \delta \in(0$, $\left.\lambda^{*}\right)$ for small $\lambda^{*}>0$ when $q=p$. Thus we may assume that $\left\langle J_{\lambda, \delta}^{\prime}\left(u_{m}, v_{m}\right),\left(u_{m}, v_{m}\right)\right\rangle \rightarrow \ell<0$. Since $\left\langle I_{\lambda, \delta}^{\prime}\left(u_{m}, v_{m}\right),\left(u_{m}, v_{m}\right)\right\rangle=0$, we conclude that $\theta_{m}=$ 0 , consequently, $I_{\lambda, \delta}^{\prime}\left(u_{m}, v_{m}\right) \rightarrow 0$. Using this information we have

$$
I_{\lambda, \delta}^{\prime}\left(u_{m}, v_{m}\right) \rightarrow c<\frac{1}{N} S_{F}^{\frac{N}{p}} \text { and } I_{\lambda, \delta}^{\prime}\left(u_{m}, v_{m}\right) \rightarrow 0,
$$

so by Lemma 2.1 the proof is completed.

Below we denote by $I_{\mathcal{N}, \delta}$ the restriction of $I_{\lambda, \delta}$ on $\mathcal{N}_{\lambda, \delta}$.

Lemma 4.3. Suppose $N \geq p^{2}, 2 \leq p \leq q<p^{*}$ and F satisfies $\left(F_{0}\right)-\left(F_{2}\right)$, let $\Lambda=\min \{\lambda *$, $\left.\lambda^{* * *}\right\}>0, \lambda, \delta \in(0, \Lambda)$, then $\operatorname{cat}_{I_{\mathcal{N}_{\lambda, \delta}}^{m_{\lambda, \delta}}}\left(I_{\mathcal{N}_{\lambda, \delta}}^{m_{\lambda, \delta}}\right) \geq \operatorname{cat}_{\Omega}(\Omega)$, where $\lambda^{*}, \lambda^{* * *}$ given by Lemmas 34 and 3.6, respectively.

Proof. Assume that $I_{\mathcal{N}_{\lambda, \delta}}^{m_{\lambda, \delta}}=A_{1} \cup A_{2} \cup \cdots \cup A_{m}$, where $A_{j}, j=1,2, \ldots, m$, are closed and contractible sets in $I_{\mathcal{N}, \delta, \delta}^{m_{\lambda, \delta}}$ i.e., there exists $h_{j} \in C\left([0,1] \times A_{j}, I_{\mathcal{N}_{\lambda, \delta}}^{m_{\lambda, \delta}}\right)$ such that

$$
h_{j}(0, z)=z, \quad h_{j}(1, z)=\vartheta, \quad \text { for all } z \in A_{j},
$$


where $\vartheta \in A_{j}$ is fixed. Consider $B_{j}=\gamma-1\left(A_{j}\right), 1 \leq j \leq m$. The sets $B_{j}$ are closed and

$$
\Omega_{r}^{-}=B_{1} \cup B_{2} \cup \cdots \cup B_{m} .
$$

We define the deformation $g_{j}:[0,1] \times B_{j}$ by setting

$$
g_{j}(t, \gamma)=H_{\lambda, \delta}\left(t, h_{j}(t, \gamma(\gamma))\right),
$$

for $\lambda, \delta \in(0, \Lambda)$. Note that

$$
g_{j}(0, \gamma)=H_{\lambda, \delta}\left(0, h_{j}(0, \gamma(\gamma))\right)=\frac{(\beta o \gamma)(\gamma)}{\alpha_{\lambda, \delta}}
$$

implies

$$
g_{j}(0, y)=\frac{\alpha_{\lambda, \delta} \gamma}{\alpha_{\lambda, \delta}}=y, \text { for all } y \in B_{j},
$$

and $g_{j}(1, y)=H_{\lambda, \delta}\left(1, h_{j}(1, \gamma(y))\right)=\beta\left(h_{j}(1, \gamma(y))\right)$ implies

$$
g_{j}(1, \gamma)=\beta(\vartheta) \in \Omega_{r}^{+} .
$$

Thus the sets $B_{j}$ are contractible in $\Omega_{r}^{+}$. It follows that $\operatorname{cat}_{\Omega}(\Omega)=\operatorname{cat}_{\Omega_{r}^{+}}\left(\Omega_{r}^{+}\right) \leq m$.

\section{Proof of Theorem 1.2.}

Using Lemmas 2.1, 2.2, and 3.3 we know that $c_{\lambda, \delta}, m_{\lambda, \delta}<\frac{1}{N} S_{F}^{\frac{N}{p}}$ for $\lambda, \delta \in(0, \Lambda)$. Moreover, by Lemma $4.2, I_{\mathcal{N}_{\lambda, \delta}}$ satisfies the $(P S)_{c}$ condition for all ${ }_{c}<\frac{1}{N} S_{F}^{\frac{N}{p}}$. Therefore, by Lemma 4.3, a standard deformation argument implies that, for $\lambda, \delta \in(0, \Lambda), I_{\mathcal{N}_{\lambda, \delta}}$ contains at least $\operatorname{cat}_{\Omega}(\Omega)$ critical points of the restriction of $I_{\lambda, \delta}$ on $\mathcal{N}_{\lambda, \delta}$. Now Lemma 4.1 implies that $I_{\lambda, \delta}$ has at least $\operatorname{cat}_{\Omega}(\Omega)$ critical points, and therefore at least cat ${ }_{\Omega}(\Omega)$ nontrivial solutions of (1.1). As Theorem 1.1, the obtained solutions are nonnegative in $\Omega$. The proof is completed.

\section{Acknowledgements}

The author would like to thank the referees for carefully reading this article and making valuable comments and suggestions. This study was supported by the Youth Foundation of Hubei Engineering University (No. Z2012003).

\section{Competing interests}

The author declares that he has no competing interests.

Received: 17 November 2011 Accepted: 28 February 2012 Published: 28 February 2012

\section{References}

1. Brezis, H, Nirenberg, L: Positive solutions of nonlinear elliptic equations involving critical Sobolev exponents. Comm Pure Appl Math. 36, 437-477 (1983). doi:10.1002/cpa.3160360405

2. Rey, O: A multiplicity results for a variational problem with lack of compactness. Nonlinear Anal. 13(10):1241-1249 (1989). doi:10.1016/0362-546X(89)90009-6

3. Alves, CO, Ding, YH: Multiplicity of positive solutions to a $p$-Laplacian equation involving critical nonlinearity. J Math Anal Appl. 279(2):508-521 (2003). doi:10.1016/S0022-247X(03)00026-X

4. Ding, L, Xiao, SW: Multiple positive solutions for a critical quasilinear elliptic system. Nonlinear Anal. 72, $2592-2607$ (2010). doi:10.1016/j.na.2009.11.007

5. Hsu, TS: Multiple positive solutions for a critical quasilinear elliptic system with concave-convex nonlinearities. Nonlinear Anal. 71(7-8):2688-2698 (2009). doi:10.1016/j.na.2009.01.110

6. Shen, Y, Zhang, JH: Multiplicity of positive solutions for a semilinear $p$-Laplacian system with Sobolev critical exponent. Nonlinear Anal. 74, 1019-1030 (2011). doi:10.1016/j.na.2010.06.066

7. Han, PG: The effect of the domain topology on the number of positive solutions of elliptic systems involving critical Sobolev exponents. Houston J Math. 32, 1241-1257 (2006)

8. Filho, DCM, Souto, MAS: Systems of $p$-Laplacian equations involving homogeneous nonlinearities with critical Sobolev exponent degrees. Comm Partial Diff Equ. 24, 1537-1553 (1999). doi:10.1080/03605309908821473 
9. Barbosa, ER, Montenegro, M: Nontrivial solutions for critical potential elliptic systems. J Diff Equ. 250, 3398-3417 (2011). doi:10.1016/j.jde.2011.01.001

10. Alves, CO, Filho, DCM, Miyagaki, OH: Multiple solutions for an elliptic system on bounded or unbounded domains. Nonlinear Anal. 56, 555-568 (2004). doi:10.1016/j.na.2003.10.004

11. Kang, DS, Peng, SJ: Existence and asymptotic properties of solutions to elliptic systems involving multiple critical exponents. Sci China Math. 54, 243-256 (2011). doi:10.1007/s11425-010-4131-3

12. Figueiredo, GM, Furtado, MF: Multiple positive solutions for a quasilinear system of Schröodinger equations. NoDEA Nonlinear Diff Equ Appl. 15, 309-333 (2008). doi:10.1007/s00030-008-7051-y

13. Lü, DF: Multiple solutions for a class of biharmonic elliptic systems with Sobolev critical exponent. Nonlinear Anal. $\mathbf{7 4}$, 6371-6382 (2011). doi:10.1016/j.na.2011.06.018

14. Chu, CM, Tang, CL: Existence and multiplicity of positive solutions for semilinear elliptic systems with Sobolev critical exponents. Nonlinear Anal. 71, 5118-5130 (2009). doi:10.1016/..na.2009.03.080

15. Adriouch, K, El Hamidi, A: The Nehari manifold for systems of nonlinear elliptic equations. Nonlinear Anal. 64, 2149-2167 (2006). doi:10.1016/j.na.2005.06.003

16. Lü, DF, Xiao, JH: Multiple solutions for weighted nonlinear elliptic system involving critical exponents. Math Comput Model. 55, 816-827 (2012). doi:10.1016/j.mcm.2011.09.007

17. Ribeiro, B: The Ambrosetti-Prodi problem for gradient elliptic systems with critical homogeneous nonlinearity. J Math Anal Appl. 363, 606-617 (2010). doi:10.1016/.jmaa.2009.09.048

18. Willem, M: Minimax Theorem. Birkhäuser, Boston (1996)

19. Brezis, $H$, Lieb, E: A relation between pointwise convergence of functions and convergence of functionals. Proc Am Math Soc. 88, 486-490 (1983)

20. Ghoussoub, N, Yuan, C: Multiple solutions for quasi-linear PDEs involving the critical Sobolev and Hardy exponents. Trans Am Math Soc. 352(12):5703-5743 (2000). doi:10.1090/50002-9947-00-02560-5

21. Lions, PL: The concentration compactness principle in the calculus of variations. The limit case I Rev Mat Iberoam. 1, 145-201 (1985)

22. Furtado, MF: Multiplicity of nodal solutions for a critical quasilinear equation with symmetry. Nonlinear Anal. 63, 1153-1166 (2005). doi:10.1016/j.na.2005.06.001

doi:10.1186/1687-2770-2012-27

Cite this article as: Lü: Multiple solutions for $p$-Laplacian systems with critical homogeneous nonlinearity. Boundary Value Problems 2012 2012:27.

\section{Submit your manuscript to a SpringerOpen ${ }^{\odot}$ journal and benefit from:}

- Convenient online submission

- Rigorous peer review

- Immediate publication on acceptance

- Open access: articles freely available online

- High visibility within the field

- Retaining the copyright to your article

Submit your next manuscript at $\gg$ springeropen.com 